\title{
Fiber Loop Mirror Using a Small Core Microstructured Fiber for Strain and Temperature Discrimination
}

\author{
Ricardo M. André, Manuel B. Marques, Philippe Roy, and Orlando Frazão
}

\begin{abstract}
In this work, a fiber loop mirror for the simultaneous measurement of strain and temperature is presented. The loop mirror contains a section of a small core microstructured fiber characterized for strain and temperature sensing. Due to the small core geometry and using a small section length, the structure presents high birefringence and also intermodal interference. The spectral response of this configuration shows the presence of three interferometers. One of them corresponds to the interference of light that propagates in the fast and slow axes (group birefringence) and the others are associated with the interference of light in the two lowest order spatial modes in each of the fiber eigenaxis. These interferometers present distinct sensitivities to strain and temperature for different wavelengths.
\end{abstract}

Index Terms-Interferometers, optical fiber sensors, photonic crystal fibers.

\section{INTRODUCTION}

$\mathbf{T}$ HE fiber loop mirror (FLM) is a frequently used device for specific applications. It is very easy to fabricate considering it is formed by a splice between the output ports of a directional optical coupler. A particular FLM configuration containing a section of highly birefringent (Hi-Bi) fiber has several advantages when compared to a more traditional interferometer [1]. In optical sensing, besides the gyroscope application, it has been used for strain [2] and temperature [3] measurement, to measure the level of liquids [4] and to sense displacement [5]. At the same time, it has been used as a spectral filter for fiber Bragg grating demodulation [6]. Recently, several authors have proposed new FLM-based configurations for strain and temperature discrimination. These configurations are based on a section of Hi-Bi fiber combined with a long-period grating [7], two

Manuscript received March 28, 2010; revised April 27, 2010; accepted May 08, 2010. Date of publication May 24, 2010; date of current version July 02, 2010. This work was supported by the COST 299-Optical Fibers for New Challenges Facing the Information Society. The work of R. M. André was supported by Fundação para a Ciência e Tecnologia under the scholarship Bolsa de Integração na Investigação.

R. M. André, M. B. Marques are with INESC Porto-Instituto de Engenharia de Sistemas e Computadores do Porto, 4169-007 Porto, Portugal and also with Departamento de Física da Faculdade de Ciências, Universidade do Porto, 4169-007 Porto, Portugal.

P. Roy is with the Photonics Department, Xlim, UMR 6172 CNRS University of Limoges, 87060 Limoges, France.

O. Frazão is with INESC Porto-Instituto de Engenharia de Sistemas e Computadores do Porto, 4169-007 Porto, Portugal (e-mail: ofrazao@inescporto.pt).

Color versions of one or more of the figures in this letter are available online at http://ieeexplore.iee.org.

Digital Object Identifier 10.1109/LPT.2010.2050474

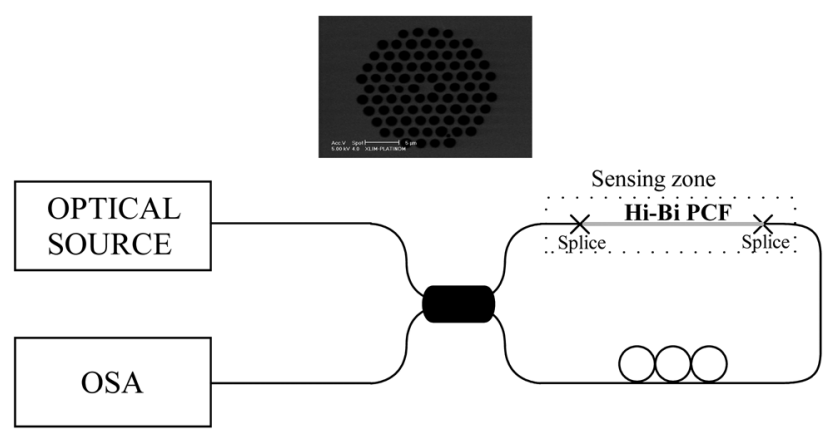

Fig. 1. Schematic diagram of the sensing system with a Hi-Bi PCF FLM (a photograph of the Hi-Bi PCF is also shown).

different types of Hi-Bi fiber [8], and two in-line Hi-Bi FLM [9]. All these configurations require two sensing structures to eliminate the temperature cross sensitivity. A possible solution is to use a pure silica section of photonic crystal fiber because the temperature sensitivity is very low [10], [11].

In this work, we present an alternative solution for the simultaneous measurement of strain and temperature using only one section of a small core microstructured fiber. The spectral response can be understood from the presence of three interferometric signatures, where two of them are associated with the interference between two spatial modes in each of the eigenaxis of the Hi-Bi fiber, and the other comes from the conventional FLM (interference of light that propagates in slow/fast axis of the Hi-Bi fiber).

\section{EXPERIMENTAL RESULTS}

\section{A. Experimental Setup}

Fig. 1 illustrates the experimental setup, which consists of an optical broadband source, a fiber-loop mirror containing a section of small core microstructured fiber, and an optical spectrum analyzer (OSA) with a maximum resolution of $50 \mathrm{pm}$. However, it could be possible to use an appropriate interrogation system, conceptually more elegant and based on the generation of quadrature phase-shifted interferometric signals through the use of dual-wavelength illumination by two discrete sources for each peak [12]. The optical source is an erbium-doped broadband source, with a central wavelength of $1550 \mathrm{~nm}$ and a spectral bandwidth of $100 \mathrm{~nm}$. The FLM is formed by a $3-\mathrm{dB}(2 \times 2)$ optical coupler with low insertion loss, an optical polarization controller (PC), and a small core microstructured fiber section with 24-cm length. The fiber 13/04 Ge was fabricated at XLim 


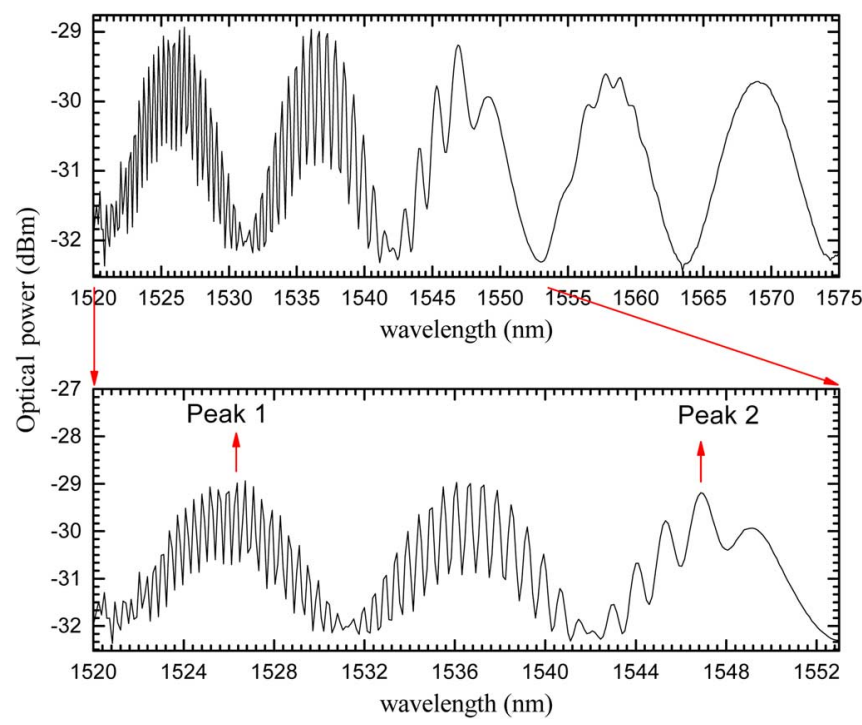

Fig. 2. Spectral response of the fiber loop sensor.

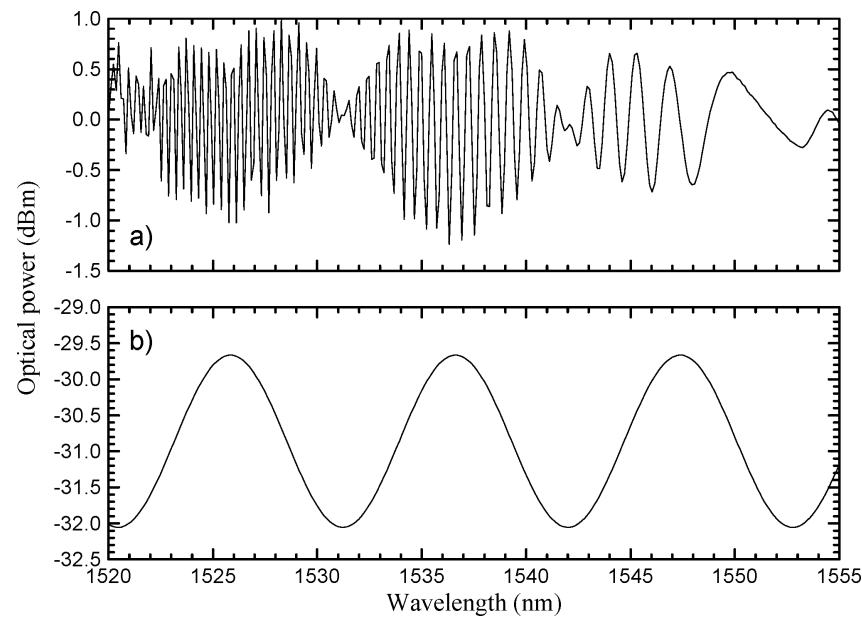

Fig. 3. Spectral response of the signals in Fig. 2 after filtering processing.

(Limoges) and presents 5 layers with 84 holes. The core diameter is $1.5 \mu \mathrm{m}$ and has a central doped region with a diameter of $1 \mu \mathrm{m}$. The diameter of the holes is $\sim 1.8 \mu \mathrm{m}$ and the pitch is $\sim 2.25 \mu \mathrm{m}$. Due to the small core and the asymmetry of the holes region, this geometry presents low birefringence (Hi-Bi), with a group birefringence value of $\sim 9.6 \times 10^{-7}$.

\section{B. Results}

Fig. 2 presents the spectral response of the sensing structure. Two fringe patterns are clearly observed. Peaks 1 and 2 are the ones used for the analysis (any two peaks well apart are appropriated) when the sensing head is affected by a particular measurand.

Fig. 3 helps us to understand the origin of the interference terms. The data in Fig. 3(a) is obtained from the data of Fig. 2 through numerically subtracting the fitted response of the Hi-Bi FLM, presented in Fig. 3(b).

The fringe pattern in Fig. 3(b) comes from the interference between light that propagates in the two polarization modes of the Hi-Bi fiber, as can be confirmed in view of the agreement of

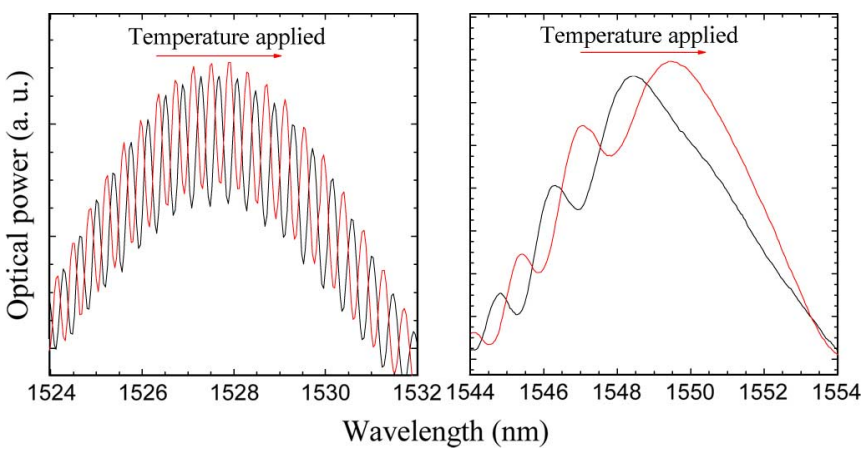

Fig. 4. Spectral response of the signals when the temperature is applied.

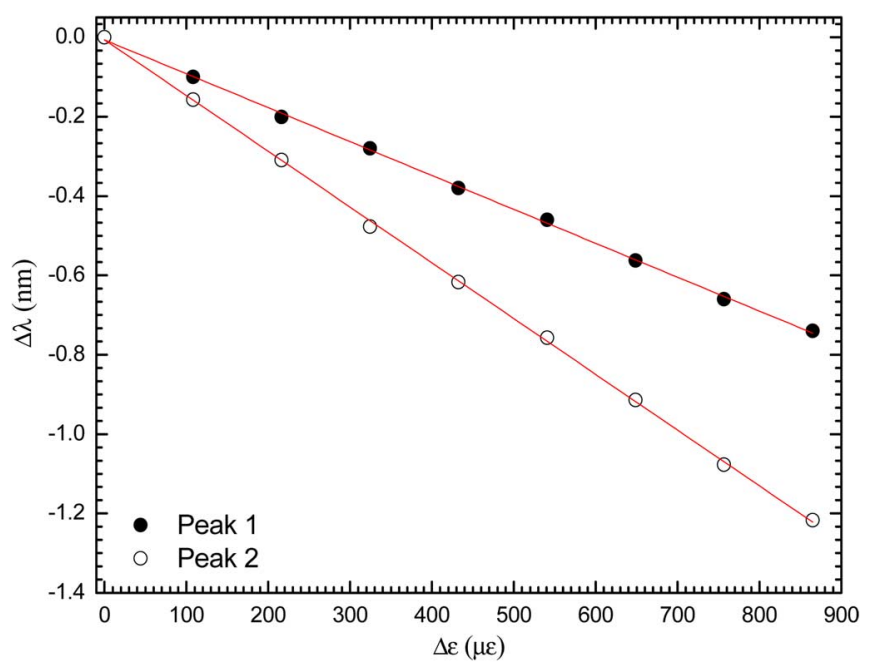

Fig. 5. Strain response of the sensing head.

the measured spectral periodicity and the value calculated from the fiber birefringence and its length. The fringe pattern shown in Fig. 3(a), with much smaller periodicity, comes from the interference between the two modes, for each polarization, supported by the core structure (which has a central region doped with germanium surrounded by a ring of pure silica). The refractive index difference between these two regions $\left(\sim 10^{-3}\right)$ is compatible with the periodicity observed (the small fringe amplitude - compared with the one of Fig. 3(b) - is an indication of the low power that propagates in the ring region). Because there is a fringe pattern of this type for each polarization, they beat together resulting in the observed envelope modulation with a periodicity close to the one associated with the interference of the light that propagates in distinct polarization axis. The chirp behavior evident in Fig. 3(a) is certainly associated to different chromatic dispersion dependences of the two core spatial modes. Fig. 4 presents an example of the pattern fringe variation when the temperature is applied.

The sensing head was characterized in strain (temperature constant) and temperature (strain constant). Fig. 5 shows the different responses of the sensing head when strain is applied. A linear blue shift is observed. On the other hand, Fig. 6 gives the results relative to temperature, where now there is a red shift. When compared with the observed behavior in conventional Hi-Bi fibers [13], the strain result is similar to what happens when the birefringence is dominated by geometric effects. The 


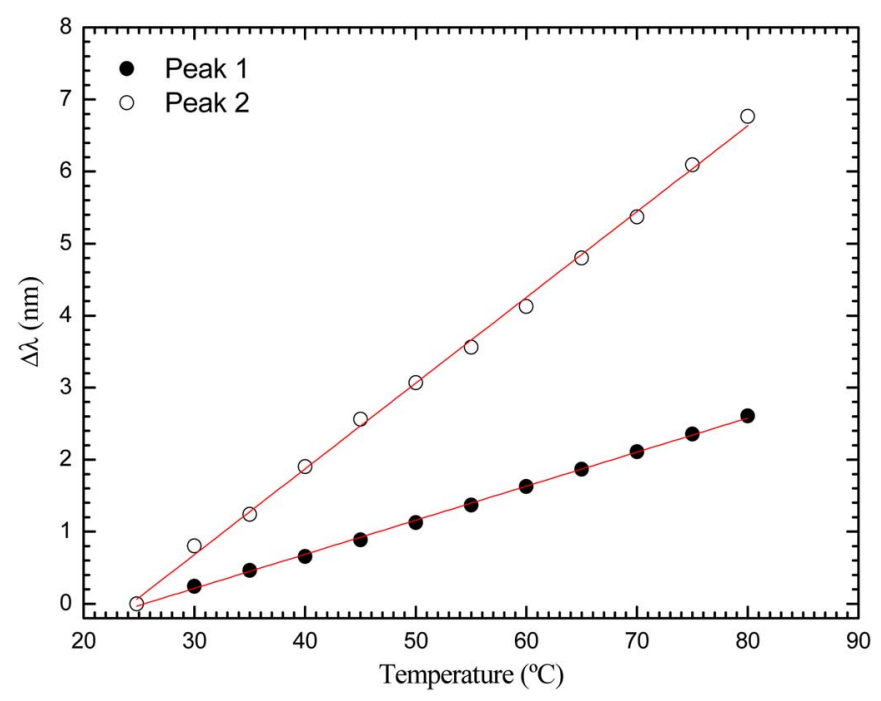

Fig. 6. Temperature response of the sensing head.

opposite effect in temperature is due to an increase in phase difference of the two modes when the temperature is applied. As the fiber length is the same for the two modes, it is required that the effective index difference between the two modes increases. As can be observed, peaks 1 and 2 present different sensitivities for the strain and temperature (see Figs. 5 and 6). This difference is expected because the pattern fringe periodicity depends strongly on the wavelength. For the same phase variation, as the pattern fringe period is different, peak 2 presents more sensitivity than peak 1 since the displacement of this fringe is higher (see Fig. 4). With these results, a well-conditioned system of two equations for $\Delta T$ and $\Delta \varepsilon$ can be written which, from the slopes indicated in Figs. 4 and 5, is given by ( $\Delta \lambda$ in picometer and $\Delta T, \Delta \varepsilon$ in degrees and microstrain, respectively)

$$
\left[\begin{array}{c}
\Delta T \\
\Delta \varepsilon
\end{array}\right]=-\frac{1}{34.9}\left[\begin{array}{cc}
1.41 & -0.85 \\
-119 & 47.3
\end{array}\right]\left[\begin{array}{l}
\delta \lambda_{\text {peak } 1} \\
\delta \lambda_{\text {peak } 2}
\end{array}\right] .
$$

The quantitative performance of this sensing structure was evaluated when the sensing head undertook strain variations in a range of $800 \mu \varepsilon$ at a fixed temperature $\left(\Delta T=55{ }^{\circ} \mathrm{C}\right)$, and the other way around, i.e., temperature variations in a range of $55{ }^{\circ} \mathrm{C}$ for a specific applied strain $(\Delta \varepsilon=400 \mu \varepsilon)$. The root-mean-square (rms) deviations relative to the applied values are $\pm 1.5^{\circ} \mathrm{C}$ and $\pm 4.7 \mu \varepsilon$ for temperature and strain, respectively, which can be considered the resolutions obtained with this structure when implementing simultaneous measurement of these two parameters.

\section{CONCLUSION}

An FLM including a small core microstructured optical fiber was characterized for simultaneous measurement of strain and temperature. Due to the structure of the core, intermodal interference was observed, resulting in a complex channeled spectrum. This effect was not explored by the research community and presents one advantage when the sensing head is used for strain and temperature discrimination. In simultaneous measurement of physical parameters, usually the configuration is a combination of two sensors in series and, in this case, the sensing head is composed only by a section fiber. The fringe pattern is strongly dependent of the wavelength and when the sensing head is subjected to a strain or temperature, a different response was obtained. This effect was explored to discriminate both parameters with resolutions of $\pm 1.5^{\circ} \mathrm{C}$ and $\pm 4.7 \mu \varepsilon$ for temperature and strain, respectively. However, this sensing head can be also used to separate three parameters combining the two peaks used in this experience and the other pattern fringe obtained by the birefringence of the fiber. Through optimization of the fiber design, a substantial reduction of its length can be obtained.

\section{REFERENCES}

[1] D. Mortimore, "Fiber loop reflectors," J. Lightw. Technol., vol. 6, no. 7, pp. 1217-1224, Jul. 1988

[2] M. Campbell, G. Zheng, A. S. Holmes-Smith, and P. A. A. Wallace, "Frequency-modulated continuous wave birefringent fiber-optic strain sensor based on a Sagnac ring configuration," Meas. Sci. Technol., vol. 10, pp. 218-224, 1999.

[3] A. N. Starodumov, L. A. Zenteno, D. Monzon, and E. De La Rosa, "Fiber Sagnac interferometer temperature sensor," Appl. Phys. Lett., vol. 70, no. 1, pp. 19-21, 1997.

[4] D. Bo, Z. Qida, L. Feng, G. Tuan, X. Lifang, L. Shuhong, and G. Hong, "Liquid-level sensor with a high-birefringence-fiber loop mirror," Appl. Opt., vol. 45, no. 30, pp. 7767-7771, 2006.

[5] Y. Liu, B. Liu, X. Feng, W. Zhang, G. Zhou, S. Yuan, G. Kai, and X. Dong, "High-birefringence fiber loop mirrors and their applications as sensors," Appl. Opt., vol. 44, no. 22, pp. 2382-2390, 2005.

[6] S. Chung, J. Kim, B.-A. Yu, and B. Lee, "A fiber Bragg grating sensor demodulation technique using a polarization maintaining fiber loop mirror," IEEE Photon. Technol. Lett., vol. 13, no. 12, pp. 1343-1346, Dec. 2001.

[7] O. Frazão, L. M. Marques, S. Santos, J. M. Baptista, and J. L. Santos, "Simultaneous measurement for strain and temperature based on a long period grating combined with a high-birefringence fiber loop mirror," IEEE Photon. Technol. Lett., vol. 18, no. 22, pp. 2407-2409, Nov. 15, 2006.

[8] G. Sun, D. S. Moon, and Y. Chung, "Simultaneous temperature and strain measurement using two types of high-birefringence fibers in Sagnac loop mirror," IEEE Photon. Technol. Lett., vol. 19, no. 24, pp. 2027-2029, Dec. 15, 2007.

[9] O. Frazão, J. M. Baptista, and J. L. Santos, "Strain and temperature discrimination using concatenated Hi-Bi fiber loop mirrors," IEEE Photon. Technol. Lett., vol. 19, no. 16, pp. 1260-1262, Aug. 15, 2007.

[10] C.-L. Zhao, X. Yang, C. Lu, W. Jin, and M. S. Demokan, "Temperatureinsensitive interferometer using a highly birefringent photonic crystal fiber loop mirror," IEEE Photon. Technol. Lett., vol. 16, no. 11, pp. 2535-2537, Nov. 2004.

[11] H. Y. Tam and X. Dong, "Temperature-insensitive strain sensor with polarization-maintaining photonics crystal fiber based on Sagnac interferometer," Appl. Opt., vol. 90, no. 15, pp. 151113-1-151113-3, 2007.

[12] O. B. Wright, "Stabilized dual-wavelength fiber-optic interferometer for vibration measurement," Opt. Lett., vol. 16, no. 1, pp. 56-58, 1991.

[13] O. Frazão, J. M. Baptista, and J. L. Santos, "Recent advances in highbirefringence fibre loop mirror sensors," MDPI Sensors J., vol. 7, no. 11, pp. 2970-2983, 2007. 